EUROPEAN ORGANIZATION FOR NUCLEAR RESEARCH

CERN - AB Division

CERN-AB-2003-030 ABP

\title{
2002 Nonlinear Optics Measurements and Modelling for the CERN SPS
}

\author{
G. Arduini, R. Tomas, F. Zimmermann (CERN) \\ A. Faus-Golfe (IFIC-Univ. de Valencia) \\ N. Iida (KEK)
}

\begin{abstract}
In 2002, nonlinear chromaticity, coupling, amplitude-dependent detuning, chromatic phase advance, resonance driving terms, and off-energy orbits were measured in the CERN SPS at $26 \mathrm{GeV} / \mathrm{c}$. The optics model has been updated by adjusting the strengths of nonlinear field errors in the dipoles and quadrupoles, so as to reproduce the measured nonlinear chromaticity. We compare the field errors deduced in 2002, measuring over a larger momentum range, with those found in 2001 and 2000 . The resolution is improved by averaging over all turn-by-turn position monitors in the ring instead of using a single dedicated pick up ('tune meter'). Computations using two different optics codes, MAD and SAD, indicate the sensitivity to matching algorithm and magnet representation. To validate the nonlinear optics model, the predicted tune shifts with transverse amplitude, driving terms of low-order resonance, etc., are compared with direct measurements.
\end{abstract}

Presented at PAC 2003, Portland, Oregon, USA

from 12 to 16 May 2003

Geneva, Switzerland

22 May 2003 


\title{
2002 Nonlinear Optics Measurements and Modelling for the CERN SPS
}

\author{
G. Arduini, R. Tomas, F. Zimmermann, CERN, Geneva, Switzerland; \\ A. Faus-Golfe, IFIC-Univ. de Valencia, Spain; N. Iida, KEK, Japan
}

\section{Abstract}

In 2002, nonlinear chromaticity, coupling, amplitudedependent detuning, chromatic phase advance, resonance driving terms, and off-energy orbits were measured in the CERN SPS at $26 \mathrm{GeV} / c$. The optics model has been updated by adjusting the strengths of nonlinear field errors in the dipoles and quadrupoles, so as to reproduce the measured nonlinear chromaticity. We compare the field errors deduced in 2002, measuring over a larger momentum range, with those found in 2001 and 2000. The resolution is improved by averaging over all turn-by-turn position monitors in the ring instead of using a single dedicated pick up ('tune meter'). Computations using two different optics codes, MAD and SAD, indicate the sensitivity to matching algorithm and magnet representation. To validate the nonlinear optics model, the predicted tune shifts with transverse amplitude, driving terms of low-order resonance, etc., are compared with direct measurements.

\section{INTRODUCTION}

Given the tight emittance budget for the SPS as LHC injector, a precise model of the nonlinear SPS optics is desirable, especially at the injection momentum of 26 $\mathrm{GeV} / c$. Since 2000 we are pursuing an experimental program aimed at establishing a fast and reliable procedure by which the SPS optics model can be updated from a fast measurement. Simultaneously, we are exploring experimental techniques that will be important for the commissioning of the LHC itself. The precursor studies of 2000 and 2001 were reported in [1] and an application to the full SPS cycle in [2]. The optics model is based on the measured nonlinear chromaticity. In 2002 we explored its sensitivity to the momentum range and to the choice of tune signal. We also compared model predictions and measurements locally, as a function of position around the ring, and we applied two different optics codes to cross-check the results. In this paper we highlight these new developments.

\section{NONLINEAR CHROMATICITY}

In the SPS, the measured betatron tunes, $Q_{x}$ and $Q_{y}$, show a strongly nonlinear dependence on the relative momentum offset, $\delta$. The latter is varied by changing the rf frequency. The two measurements of 2002 are displayed in Fig. 1. The different pictures show two different beam signals, i.e., either from the 'tune meter' pick-up or from about $2 \times 110$ beam-position monitors (BPMs), together with various fits and model predictions. The linear, quadratic and cubic components of the two chromaticities are defined as

$$
Q_{x, y}=Q_{0 x, y}+Q_{x, y}^{\prime} \delta+\frac{1}{2} Q_{x, y}^{\prime \prime} \delta^{2}+\frac{1}{6} Q_{x, y}^{\prime \prime \prime} \delta^{3},
$$
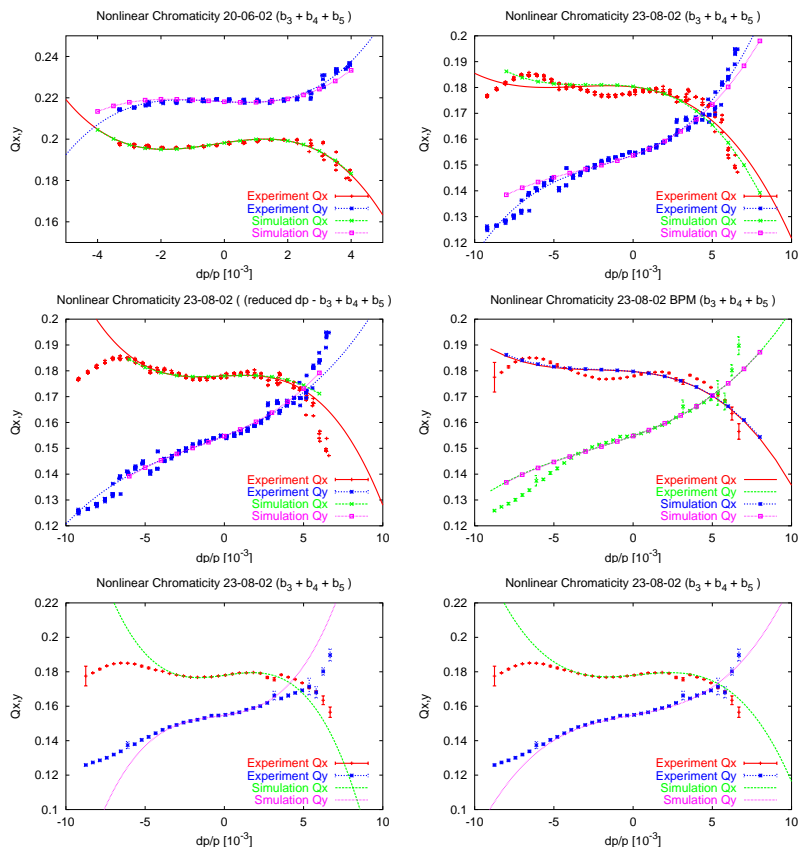

Figure 1: Measured and predicted horizontal and vertical tune versus momentum offset, for the experiment on $20^{t h}$ June 2002 (top left) and on $23^{\text {rd }}$ August 2002 (others); with fit over $\delta=-0.009 / 0.007$ (top right); $\delta=-0.005 / 0.005$ (centre left); again $\delta=-0.009 / 0.007$ (centre right); $\delta=$ $-0.003 /+0.003$ (bottom left); $\delta=-0.005 /+0.0055$ (bottom right). The bottom row was matched for $K_{5 a} \neq K_{5 b}$ using SAD; the other four with $K_{5 a}=K_{5 b}$ using MAD. The data in the first three plots are from a dedicated application ('tune meter'), the last three were obtained by averaging over all BPMs.

and can be obtained by a fit. Table 1 summarizes the coefficients found in 2002. The table also indicates the $\delta$ interval.

To construct an SPS optics model with identical nonlinear chromatic behavior, we add sextupolar and decapolar field errors in the two types of dipole magnets (called MBB and MBA) and octupole errors in the two types of quadrupoles [1]. We then determine the strength of these field errors so as to achieve best agreement with the 6 linear and nonlinear chromatic coefficients. We distinguish between the sextupole errors in MBB and MBA, but often assume that the decapole errors are the same. This is done to avoid a degeneracy problem when fitting with MAD. In SAD fits, we kept the distinction between the two types of errors, which we denote by $K_{5 a}$ and $K_{5 b}$ (a similar notation is used for the other types of errors). Our procedure assumes that all magnets have the same systematic error and that the nonlinear fields arise from the regular cells. 
Table 1: Linear and nonlinear chromatic coefficients obtained from a polynomial fit of the measured betatron tune as a function of $\mathrm{rf}$ frequency.

\begin{tabular}{|l|r|r|r|r|r|}
\hline 2002 MDs & $Q_{0 x}$ & $Q_{x}^{\prime}$ & $Q_{x}^{\prime \prime}$ & $Q_{x}^{\prime \prime \prime}$ & $R_{x}^{2}$ \\
$\mp$ fit & $Q_{0 y}$ & $Q_{y}^{\prime}$ & $Q_{y}^{\prime \prime}$ & $Q_{y}^{\prime \prime \prime}$ & $R_{y}^{2}$ \\
& & & {$\left[10^{2}\right]$} & {$\left[10^{5}\right]$} & \\
\hline \hline $20 / 06 / 2002$ & 0.1982 & 2.3 & -6.0 & -18.0 & 0.87 \\
$-0.003 / 0.004$ & 0.2181 & -0.8 & 6.0 & 18.0 & 0.95 \\
\hline \hline $23 / 08 / 2002$ & 0.1804 & -0.7 & -6.0 & -2.4 & 0.76 \\
$-0.009 / 0.007$ & 0.1537 & 2.2 & 4.0 & 2.4 & 0.98 \\
\hline & 0.1780 & 0.3 & -0.6 & -3.0 & 0.81 \\
$-0.005 / 0.005$ & 0.1547 & 2.4 & 2.0 & 1.20 & 0.96 \\
\hline \hline $23 / 08 / 2002$ & 0.1797 & -0.5 & -3.4 & -1.1 & 0.75 \\
$-0.009 / 0.007$ & 0.1574 & 2.2 & 1.8 & 1.8 & 0.98 \\
$(\mathrm{BPMs})$ & & & & & \\
\hline
\end{tabular}

In Table 2 we compare the 2002 field errors with those of the two previous years. In 2000 a single measurement was performed, in the later years several. The error quoted reflects the rms spread over the measurements of one year. The last column gives the average and spread over all years. We observe a significant fluctuation, which is partly caused by different SPS cycles, and, thus, related to actual changes in the remanent fields. However, a large variation is observed even between measurements of the same year and for the same cycle.

Table 2: Matched multipole components for linear and nonlinear chromaticity measurements in 2000, 2001 [1] and 2002 using MAD, and assuming $K_{5 a}=K_{5 b}$ (三 $K_{5}$ ).

\begin{tabular}{|l|r|r|r|}
\hline element & dipoles & quadr. & dipoles \\
\hline MDs & $K_{3 a}$ & $K_{4 f}$ & $K_{5}$ \\
& $K_{3 b}$ & $K_{4 d}$ & \\
\hline units & $10^{-3}\left[\mathrm{~m}^{-2}\right]$ & $10^{-1}\left[\mathrm{~m}^{-3}\right]$ & {$\left[\mathrm{m}^{-4}\right]$} \\
\hline \hline $\mathbf{2 0 0 0}$ & 1.4 & 0.8 & -5.8 \\
& -0.8 & -2.6 & \\
\hline $\mathbf{2 0 0 1}$ & $1.2 \pm 0.3$ & $1.1 \pm 0.1$ & $-20 \pm 4$ \\
& $-3.0 \pm 0.5$ & $-0.8 \pm 2.3$ & \\
\hline $\mathbf{2 0 0 2}$ & $0.6 \pm 0.2$ & $-0.2 \pm 0.1$ & $-12 \pm 12$ \\
& $-2.5 \pm 0.1$ & $-1.5 \pm 0.8$ & \\
\hline \hline total & $1.0 \pm 0.3$ & $0.5 \pm 0.5$ & $-12 \pm 6$ \\
& $-2.1 \pm 0.8$ & $-1.6 \pm 0.8$ & \\
\hline
\end{tabular}

A possible reason for the variation are differences in the momentum range. This is supported by the various curves and pictures in Fig. 1 (the last five pictures of which show the same experimental data fitted over different momentum ranges with two different programs), and also by Table 3, which confirms a strong dependence of the fitted chromatic coefficients on the momentum interval considered. Table 4 displays the associated multipole errors. In particular, the higher-order errors $K_{4 d}, K_{5 a}$ and $K_{5 d}$ are not well constrained and vary dramatically with the fit range.
Table 3: Linear and nonlinear chromatic coefficients obtained from a polynomial fit to the experimental data on $23^{r d}$ August 2002, for different $\delta$ ranges.

\begin{tabular}{|l|r|r|r|r|}
\hline $\begin{array}{l}\mathbf{2 3 / 0 8 / 0 2} \\
\mp \delta \text { fit }\end{array}$ & $Q_{0 x}$ & $Q_{x}^{\prime}$ & $Q_{x}^{\prime \prime}$ & $\begin{array}{r}Q_{x}^{\prime \prime \prime} \\
Q_{0 y}^{\prime \prime}\end{array}$ \\
& & $Q_{y}^{\prime}$ & $\begin{array}{r}Q_{y}^{\prime \prime} \\
Q_{y}^{\prime \prime \prime} \\
{\left[10^{2}\right]}\end{array}$ \\
\hline \hline$-0.003 /$ & 0.1780 & 1.27180 & 0.7 & -8.7 \\
+0.003 & 0.1547 & 1.40673 & 4.3 & 7.7 \\
\hline$-0.005 /$ & 0.1779 & 1.04863 & 1.2 & -5.4 \\
+0.005 & 0.1548 & 1.78427 & 2.2 & 3.0 \\
\hline$-0.0045 /$ & 0.1779 & 0.89959 & 1.9 & -4.9 \\
+0.005 & 0.1549 & 1.68147 & -0.5 & 2.7 \\
\hline$-0.005 /$ & 0.1779 & 0.88922 & 1.9 & -4.8 \\
+0.0055 & 0.1549 & 1.66710 & 0.6 & 3.4 \\
\hline
\end{tabular}

Table 4: Matched multipole components from linear and nonlinear chromaticity measurements on $23^{r d}$ August 2002 for different fit intervals of $\delta$ and $K_{5 a} \neq K_{5 b}$.

\begin{tabular}{|l|r|r|r|}
\hline element & dipoles & quadrupoles & dipoles \\
\hline $\mathbf{2 3 / 0 8 / 0 2}$ & $K_{3 a}$ & $K_{4 f}$ & $K_{5 a}$ \\
$\mp \delta$ fit & $K_{3 b}$ & $K_{4 d}$ & $K_{5 b}$ \\
\hline units & $10^{-3}\left[\mathrm{~m}^{-2}\right]$ & $10^{-1}\left[\mathrm{~m}^{-3}\right]$ & {$\left[\mathrm{m}^{-4}\right]$} \\
\hline \hline$-0.003 /$ & 0.841 & 0.38 & -10.0 \\
+0.003 & -2.61 & -1.76 & -29.9 \\
\hline$-0.005 /$ & 0.831 & 0.39 & -8.6 \\
+0.005 & -2.71 & -0.84 & -6.1 \\
\hline$-0.0045 /$ & 0.782 & 0.42 & -7.78 \\
+0.005 & -2.65 & 0.39 & -5.77 \\
\hline$-0.005 /$ & 0.778 & 0.44 & -6.55 \\
+0.0055 & -2.65 & -0.13 & -10.74 \\
\hline
\end{tabular}

\section{VALIDATION}

Once the nonlinear optics is constructed, we compare its predictions for various optics parameters (other than chromaticity) with direct measurements. A primary test is the detuning with amplitude. Figure 2 shows an example, where the agreement is good for the vertical tune. The residual discrepancy for the horizontal tune decreases if the $\delta$ range of the fit is narrowed. Hence, the global behavior of the SPS optics is fairly well reproduced by our model.
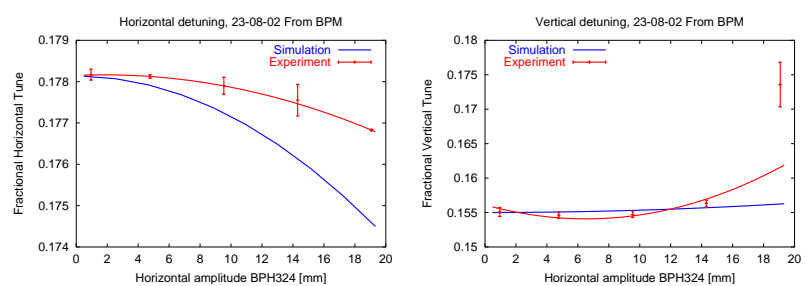

Figure 2: Predicted tune shift vs. horizontal amplitude in $\mathrm{mm}\left(\right.$ at $\beta_{x} \approx 100 \mathrm{~m}$ ) compared with measurement using BPM data.

Figure 3 displays measurements and predictions for 
the off-momentum 3rd-oder resonance driving term $f_{3000}$ $[5,6]$ at all BPMs around the SPS. The prediction in the left picture includes the fitted multipole errors; in the right picture it does not. The agreement is better, but not perfect, if the fitted multipole errors are taken into account, which supports our hypothesis of distributed errors.
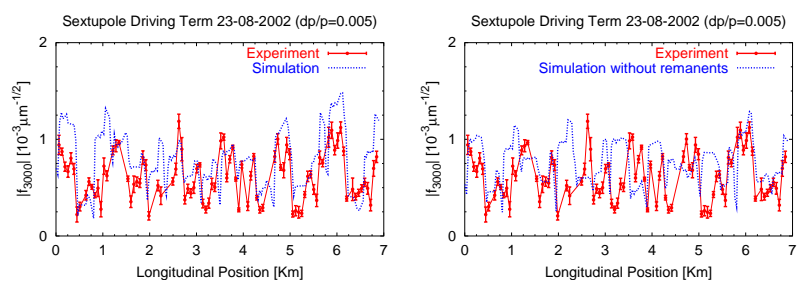

Figure 3: Amplitude of $f_{3000}$ versus the longitudinal position measured at $\delta=0.005$, and the prediction with (left) and without (right) the fitted multipole errors.

For the octupolar driving term $f_{4000}^{\prime}[5,6]$, the simulation (including the fitted multipole errors) and measurement are compared in the left picture of Fig. 4. Except for the first experimental peak at the beginning of the ring, the agreement between model and experiment is consistent within the error bars.
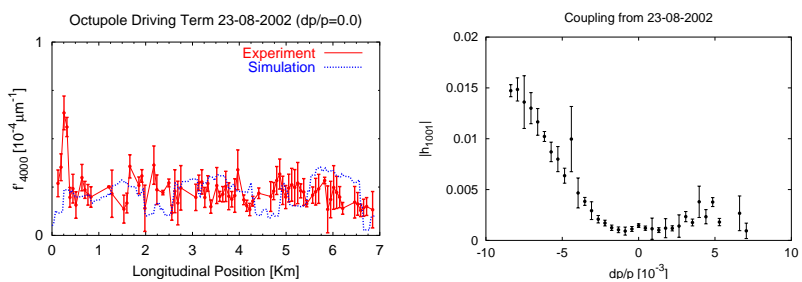

Figure 4: Left: measured and predicted amplitude of the term $f_{4000}^{\prime}$ versus longitudinal position at $\delta=0.0$; right: measured coupling resonance driving term versus $\delta$.

From turn-by-turn beam positions recorded after horizontal kick excitation, we can also infer the horizontal betatron phase advance between BPMs. Measuring for two different values of $\delta$ and computing the difference at every BPM yields the chromatic phase advance around the ring. Figure 5 compares this with the model prediction. Again, including the fitted errors improves the agreement.
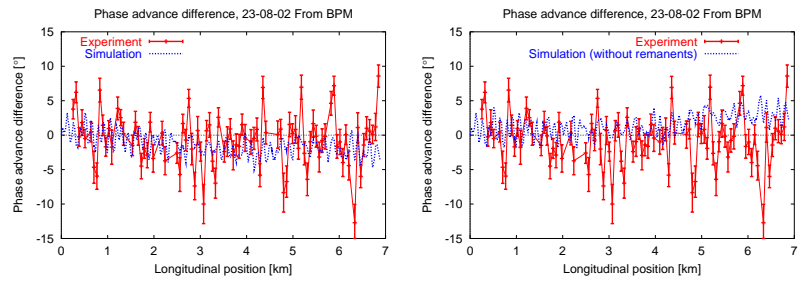

Figure 5: Measured and predicted phase advance differences for $\delta=0.0$ and 0.005 vs. longitudinal position with (left) and without (right) the fitted multipole errors.

\section{COUPLING \& ORBIT}

We also measured the strength of the linear coupling as a function of the energy deviation. The coupling was inferred from the secondary spectral lines, as described in [5]. The result is shown in the right picture of Fig. 4. For negative $\delta$, the dependence is almost linear, but for positive $\delta$ the coupling stays small and fairly constant. If this dependence is caused by a progressive distortion of the vertical closed orbit, when the vertical tune approaches the integer resonance as a result of the momentum deviation, the vertical orbit should roughly vary as $1 /\left[\sin \left(\pi Q_{y}\right)(1+\delta)\right]$. This is indeed the case, as shown in the left picture of Fig. 6 for a single BPM. Plotting the fitted slope (i.e., the slope of the line in the left picture) for all the BPMs of the ring, we obtain the right picture. The number of oscillations coincides with the integer tune.
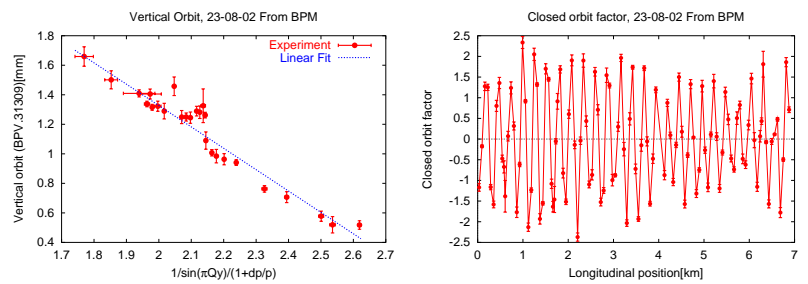

Figure 6: Left: vertical closed orbit vs. $1 /\left[\sin \left(\pi Q_{y}\right)(1+\right.$ $\delta)$ ] at BPV.31309; right: fitted slope ("closed orbit factor") at all BPMs versus the longitudinal location.

\section{CONCLUSIONS}

As in previous years, in 2002 the nonlinear optics model of the SPS was updated from the measured nonlinear chromaticity. Compared with earlier measurements the momentum range was widely increased. We simultaneously sampled the turn-by-turn beam position at all BPMs around the ring, which gives a better resolution and also provides many complementary optics informations. And we applied two different optics codes for the fits. The nonlinear multipole components obtained using MAD and SAD are consistent, except for one of the two octupole errors $\left(K_{4 d}\right)$ and for the decapoles. These are the multipoles which also are most sensitive to the momentum range of the fit. To validate the model, its predictions were compared with various complementary optics measurements, such as the detuning with amplitude, resonance driving terms, and chromatic phase advance. In general, the fitted nonlinear field errors improve the agreement between predictions and measurements, even locally around the ring.

\section{REFERENCES}

[1] G. Arduini, et al., CERN SL Note 2001-030 MD; SL Note 2001-049 MD; and Proc. EPAC 2002, Paris, p. 1220.

[2] J. Wenninger, CERN SL-Note-2002-041 OP.

[3] H. Grote, F.C. Iselin, CERN/SL/90-13 (AP).

[4] K. Oide, et al., http://acc-physics.kek.jp/SAD/sad.html.

[5] R. Tomas, PhD thesis, U. de Valencia 2003.

[6] R. Bartolini, F. Schmidt, Part. Acc. 59, p. 93 (1998). 